\title{
Chikungunya virus and dengue virus coinfection: a case report from Bangladesh
}

\author{
Muhammad Abdur Rahim ${ }^{1}$, Shahana Zaman', Samira Rahat Afroze ${ }^{3}$, Hasna Fahmima Haque, \\ Farhana Afroz ${ }^{3}$, Tabassum Samad ${ }^{1}$, Khwaja Nazim Uddin ${ }^{3}$ \\ ${ }^{1}$ Department of Nephrology, BIRDEM and IMC, Dhaka, Bangladesh; ${ }^{2}$ Department of Cardiology, \\ NICVD, Dhaka, Bangladesh; ${ }^{3}$ Department of Internal Medicine, BIRDEM and IMC, Dhaka, Bangladesh
}

\begin{abstract}
A case of concurrent chikungunya virus and dengue virus infection is reported here. The patient presented with fever and generalized body ache. Diagnostic work-up revealed chikungunya-dengue co-infection. Dengue is endemic in Bangladesh while chikungunya is a recently emerging infection. As both the viruses are transmitted by a common vector, Aedes spp., such co-infections are likely to increase in coming years.
\end{abstract}

IMC J Med Sci 2018; 12(1): 42-43

\section{Introduction}

Chikungunya and dengue are two important and rapidly spreading mosquito-borne viral infections of global concern including Bangladesh. Dengue is endemic and chikungunya is an emerging infection in Bangladesh [1-3]. Since both the viruses are transmitted by Aedes mosquitoes, simultaneous or sequential infections by chikungunya and dengue viruses are not impossible. Here, we report a case of chikungunya-dengue co-infection occurring in a middle aged Bangladeshi patient.

\section{Case report}

A 50-year-old lady presented with 3-day history of high grade continued fever and generalized body ache. She suffered a 5-day long febrile illness starting 15 days ago. Since the onset of fever for the first time, she had been experiencing pain in her hands and feet. She took paracetamol tablets during febrile periods and did not seek any medical advice before presenting to our center.

On examination, patient was febrile (temperature $102^{\circ} \mathrm{F}$ ) but hemodynamically stable (pulse $92 / \mathrm{min}$, blood pressure 130/80 mm Hg). There was no rash or lymphadenopathy. Other physical examination findings were unremarkable.
Investigations revealed leucopenia [total white blood cell (WBC) count $2.5 \times 10^{9} / \mathrm{L}$ ], lower normal platelet counts $\left(150 \times 10^{9} / \mathrm{L}\right)$ and slightly raised erythrocyte sedimentation rate $\left(25 \mathrm{~mm}\right.$ in $1^{\text {st }}$ hour $)$. Both dengue nonstructural protein 1 (NS1) and immunoglobulin $\mathrm{M}$ (IgM) for chikungunya were positive by immuno-chromatographic test (Dengue NS1 by Humasis Co. Ltd., Republic of Korea; chikungunya $\operatorname{IgM} / \mathrm{IgG}$ by $\mathrm{SD}$ BIOSENSOR, Republic of Korea). A diagnosis of chikungunyadengue co-infection was made. She was treated with paracetamol and became afebrile after 5 days. Pain in feet continued even two weeks after she became afebrile and she was prescribed oral prednisolone $15 \mathrm{mg} /$ day initially, with a plan to gradually tapper off over three weeks.

\section{Discussion}

Chikungunya and dengue virus co-infections have been reported from India [4], Thailand [5], Yemen [6] and among returning travelers from Colombia [7] and Angola [8]. As both the viruses share common vector, chikungunya-dengue co-infection is likely to occur elsewhere. As chikungunya is a relatively new entity in Bangladesh [3], we did not find many cases of such co-infections, but we predict to deal increasing number of such co-

Address for Correspondence:

Dr. Muhammad Abdur Rahim, Assistant Professor, Department of Nephrology, BIRDEM General Hospital and IMC, 122 Kazi Nazrul Islam Avenue, Dhaka-1000, Bangladesh. Email: muradrahim23@yahoo.com 
infections in coming years. In our clinical practice, we dealt patients with chikungunya with evidences of past dengue infection (positive anti-dengue IgG antibody; unpublished observations).

Chikungunya and dengue viruses share many similar epidemiological and clinical characteristics. Both can cause fever, rash, body aches and pains; though arthritis is more associated with chikungunya while retro-orbital pain is frequently present in dengue $[2,3]$. Reverse-transcriptase polymerase chain reaction (RT-PCR) technology is now-a-days available; which can efficiently detect ribonucleic acids (RNAs) of chikungunya and dengue sufficiently early in disease course. Leucopenia and thrombocytopenia are common in dengue whereas lymphocytopenia and raised erythrocyte sedimentation rate favor diagnosis of chikungunya infection [2,3]. Antichikungunya antibody (IgM) and anti-dengue antibody ( $\operatorname{IgM})$ may be detected in later part of first week or at the beginning of second week. Exclusion of dengue is more important than establishing chikungunya virus infection during febrile periods, as patients may require nonsteroidal anti-inflammatory drugs (NSAIDs), which is not advocated during dengue infection [3]. Not only that, dengue has a higher mortality (0.5-3.5\%) than chikungunya $(<0.1 \%)$ and simultaneous infections by both viruses may result in serious disease though controversies exist [1].

Like any other vector borne disease, mosquito control is an important intervention to prevent dengue and chikungunya infections. Confining patients suffering from chikungunya and dengue under mosquito nets during viremic stage is an important intervention against disease transmission [3]. Creating and improving mass awareness, cleaning mosquito breeding sites as well as other public health measures are necessary to effectively reduce the burden of chikungunya and dengue infections in Bangladesh.

\section{Author's contributions}

MAR diagnosed and managed the case, collected data, did literature search and drafted the manuscript. SZ did literature search and helped in manuscript preparation. SRA, HFH, FH and TS followed up the case, collected data and edited the manuscript. KNU was the overall supervisor in diagnosing and managing the case and manuscript preparation.

\section{Competing interest}

Authors declare no conflict of interest.

\section{Funding}

None

\section{References}

1. Furuya-Kanamori L, Liang S, Milinovich G, Magalhaes RJS, Clements ACA, Hu W, et al. Co-distribution and co-infection of chikungunya and dengue viruses. BMC Infect Dis. 2016; 16: 84.

2. Ahmed JU, Rahim MA, Uddin KN. Emerging Viral Diseases. BIRDEM Med J. 2017; 7(3): 224-32.

3. Rahim MA, Uddin KN. Chikungunya: an emerging viral infection with varied clinical presentations in Bangladesh. Reports of seven cases. BMC Res Notes. 2017; 10: 410.

4. Saswat T, Kumar A, Kumar S, Mamidi P, Muduli S, Debata NK, et al. High rates of coinfection of Dengue and Chikungunya virus in Odisha and Maharashtra, India during 2013. Infect Genet Evol. 2015; 35: 134-41.

5. Laoprasopwattana K, Suntharasaj T, Petmanee $\mathrm{P}$, Suddeaugrai O, Geater A. Chikungunya and dengue virus infections during pregnancy: seroprevalence, seroincidence and maternalfetal transmission, southern Thailand, 20092010. Epidemiol Infect. 2016; 144(2): 381-88.

6. Rezza G, El-Sawaf G, Faggioni G, Vescio F, Al Ameri R, De Santis R, et al. Co-circulation of Dengue and Chikungunya Viruses, Al Hudaydah, Yemen, 2012. Emerg Infect Dis. 2014; 20(8): 1351-54.

7. Rosso F, Pacheco R, Rodríguez S, Bautista D. Co-infection by Chikungunya virus (CHIK-V) and dengue virus (DEN-V) during a recent outbreak in Cali, Colombia: Report of a fatal case. Rev Chilena Infectol. 2016; 33(4): 464-67.

8. Parreira R, Centeno-Lima S, Lopes A, Portugal-Calisto D, Constantino A, Nina J. Dengue virus serotype 4 and chikungunya virus coinfection in a traveller returning from Luanda, Angola, January 2014. Euro Surveill. 2014; 19(10).pii: 20730. 